\title{
Prediction Equations of Physical Fitness Age for Korean Adults
}

\author{
Byoung-goo Ko $\mathrm{PhD}^{1, \dagger}$, Ji-won Seo $\mathrm{MS}^{2, \dagger}$, Bong-ju Sung ${ }^{1}$, Wook Song $\mathrm{PhD}^{2,3}$, Jun Hyun Bae $\mathrm{PhD}^{2}$, Byunggul Lim MS ${ }^{2}$, \\ Parivash Jamrasi MS ${ }^{3}$ \\ ${ }^{1}$ Korea Institute of Sport Science, Seoul; ${ }^{2}$ Department of Physical Education, Seoul National University, Seoul; ${ }^{3}$ Institute on Aging, Seoul National University, Seoul, Korea
}

PURPOSE: This study aimed to develop prediction equations for estimating the physical fitness age (PFA) of Korean adults in young (19-40 years), middle (41-64 years), and old (65-80 years) age groups.

METHODS: Data from 122,842 individuals who participated in Korea National Physical Fitness Survey and National Fitness 100 from 2009 to 2014 were collected. Body composition, muscular strength, muscular endurance, flexibility, cardiorespiratory endurance, agility, power, balance, and coordination were measured. Pearson's correlation and stepwise regression analyses were used to analyze the data.

RESULTS: The equations were as follows: PFA for young males=22.321 -.088 (20-m PACER)+.317 (body mass index [BMI]); PFA for young females $=24.486-.143(20$-m PACER) $+.304(B M I)$; PFA for middle-aged males=66.644 -.044 (standing long jump) -.069 (20-m PACER) - .201 (weight) -.075 (modified sit-ups) +.269 (10-m shuttle run)+.320 (BMI); PFA for middle-aged females=66.814 -.098 (standing long jump) -.113 (modified sit-ups); PFA for older males $=84.795+.093$ (figure-of- 8 walk) -.100 (chair standing) -.122 (weight) - .102 (relative grip strength) -.060 (sit-and-reach)+.147 (3-m up-and-go); and PFA for older females $=80.577+.097$ (figureof-8 walk)+.306 (3-m up-and-go) -.280 (weight) -.088 (relative grip strength) -.069 (sit-and-reach)+.393 (BMI) -.088 (chair standing) -.011 (2-min step-in-place).

CONCLUSIONS: Our prediction equations for PFA can be used as a tool to prescribe sex- and age-appropriate exercise program and to verify the effect of the application of the exercise program by comparing pre -and post-PFA.

Key words: Fitness age, National fitness 100, Young, Middle, Old

\section{INTRODUCTION}

Chronological age (CA) is the number of years a person has lived and is a commonly used indicator to track aging [1]. Nowadays, however, many people are paying attention to biological age (BA) because not all individuals age at the same rate. BA is the progressive decrease in viability and increased vulnerability in the body over time, which ultimately leads to death [2]. Therefore, an individual's BA can be either older or younger than their CA [3]. Thus, BA has been used increasingly in aging research to measure the progress of the biological aging process as opposed to simply measuring the passage of time [4].

Previous studies have established various statistical models to estimate
BA based on cognitive age [5], physical fitness age (PFA) [6,7], perceived age $[8,9]$, work ability index [10], and frailty index [11-13], combining physical, physiological, or biochemical parameters using mathematical methods. As a result, some studies have shown that PFA has a high correlation with $\mathrm{BA}[6,7]$.

To promote physical fitness and activities of Korean citizens, Korea Ministry of Culture, Sports and Tourism introduced the National Fitness 100 project. It is a national sports promotion project that scientifically measures and evaluates physical fitness, providing customized exercise prescriptions according to the level of baseline physical fitness [14]. To provide effective exercise programs, the PFA according to the individual physical fitness level, instead of CA, should be considered. Addi-

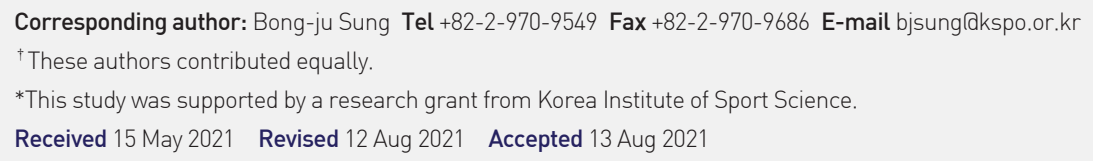


tionally, exercise not only prevents aging, but also increases muscle endurance, flexibility, and balance [15]. And muscular atrophy occurs with aging after adulthood, and is worse when physical exercise is lacking [16]. Therefore, we expect that the comparison between PFA and CA will encourage people to participate in exercise which has many benefits.

Due to the importance of PFA, several studies have been conducted to estimate PFA [17-25]. However, few studies have been scientifically rigorous, and systematic large-scale reports on a PFA estimation formula are limited. Those that do exist have limitations in that they target one sex or age group, or include only a small sample size, which limits the statistical analysis. We posit that developing prediction equations that consider age and sex using Korean participants will result in a more accurate estimate of the PFA for the Korean population.

Therefore, this study was aimed at developing prediction equations for estimating the PFA of Koreans according to young (19-40 years), middle (41-64 years), and old (65-80 years) age groups.

\section{METHODS}

\section{Participants}

The subjects were 122,842 individuals who participated in the Survey of National Physical Fitness and National Fitness 100 program from 2009 to 2014. The data was collected from 19 national fitness award centers nationwide, and outliers were excluded (Fig. 1). In accordance with previous studies on health or physical function [26-28], we divided the participants into 3 age groups, the young (19-40 years), middle (41-64 years), and old (65-80 years) age groups.

This study was approved by the institutional review board of Seoul National University (IRB No. E2007/003-006).

\section{Measurement variables}

Measurements of body mass index (body composition, BMI), maximal and relative grip strength (both muscular strength), modified sit-ups (muscular endurance), sit-and-reach (flexibility), 20-m PACER (cardiorespiratory endurance), 10-m shuttle run (agility), and standing long jump (power) were performed for the young and middle age groups. Data on BMI (body composition), 6-minute walk test and 2-minute step-in-place (both cardiorespiratory endurance), maximal and relative grip strengths (both muscular strengths), chair standing (muscular endurance), sit-andreach (flexibility), 3-m up-and-go (balance), and figure-of- 8 walk (coordination) were collected for the old age group.

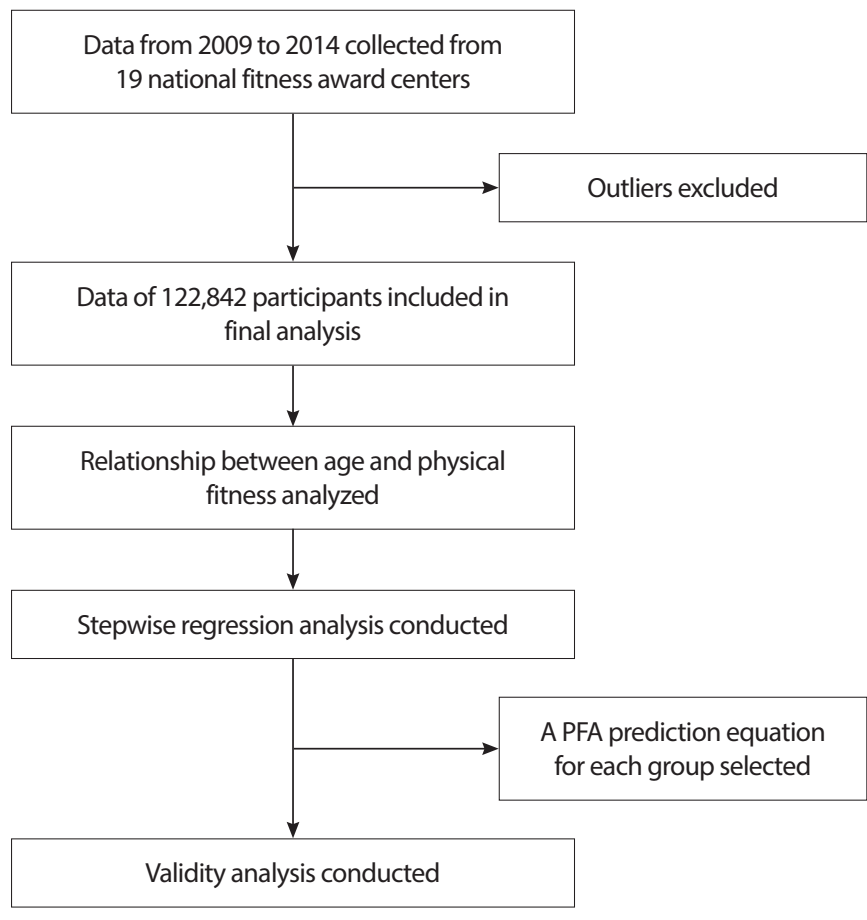

Fig. 1. Study design.

All measurements were conducted by trained physical fitness instructors and performed in accordance with the directions from The Survey of National Physical Fitness [29] and Development of National Physical Fitness Certification Standards for adults [30] and the elderly [31]. BMI is weight $(\mathrm{kg})$ divided by the square of height $\left(\mathrm{m}^{2}\right)$. Relative grip strength $(\mathrm{kg})$ is maximal grip strength $(\mathrm{kg})$ divided by weight. Modified sit-ups (number of times) was measured for 1 minute. The sit-and-reach test distance $(\mathrm{cm})$ was measured 3 times, and the highest value was recorded. The 20-m PACER (number of times) was measured by running $20 \mathrm{~m}$ back and forth with a beep for pacing. The 10-m shuttle run (seconds) was measured as the time to complete 2 round trips of $10 \mathrm{~m}$. Standing long jump $(\mathrm{cm})$ was used to measure the forward jumping distance from an initial point without any help. The 6-minute walk test (m) was used to measure the distance covered after walking for 6 minutes. The 2-minute step-in-place (number of times) was used to measure the number of steps while standing in-place for 2 minutes. Chair standing was measured (number of times) by sitting on a chair and standing up for $30 \mathrm{sec}-$ onds. The 3-m up-and-go (seconds) was measured as the time to reach a target $3 \mathrm{~m}$ away and return. For the figure-of- 8 walk (seconds), cones were set at both bottom points of an isosceles triangle with a base of 3.6 $\mathrm{m}$ and a height of $1.6 \mathrm{~m}$, and a chair was placed at the vertex point. The participants started sitting on the chair. They turned the right cone and 
returned to sit on the chair, then turned the left cone and returned to sit on the chair again. The time of completion of the 2 laps was measured.

\section{Statistical analysis}

The data were analyzed using IBM SPSS Statistics 21.0 (IBM Corp., Armonk, NY, USA). Descriptive statistical analysis, Pearson's correlation analysis, and stepwise regression analysis were conducted. Pearson's correlation analysis was used to analyze the relationship between age and physical fitness according to sex and age group. Stepwise regression analysis was applied in the development of the prediction equations according to sex and age group. Finally, 25\% of the subjects were randomly sampled to estimate their PFAs and to confirm the cross validity of the prediction equations (Fig. 1). Statistical significance was set at $p<.05$.

\section{RESULTS}

\section{Physique and physical fitness characteristics of participants}

Physique and physical fitness characteristics of the participants are presented in Table 1. In most physical fitness variables except sit-andreach, males and younger group showed better performance than females and older group. However, the old age female group was more flexible than the young age female in sit-and-reach, and the old age female walked longer distances than the male group in the 6-minute walk test.
In addition, in sit-and-reach, maximal grip strength, and relative grip strength which are the common variables of all groups, the difference between middle-aged and old age group was greater than the difference between young and middle-aged group.

\section{Analysis of relationship between age and physical fitness}

In the young age group, the analysis revealed that all physique and physical fitness variables significantly correlated with age for both sexes, followed by 20-m PACER (male $r=-.317$, female $r=-.235$ ), modified situps (male $r=-.289$, female $r=-.204$ ), and BMI (male $r=.210$, female $r=$ .182; Table 2).

In the middle-aged male group, all physique and physical fitness variables significantly correlated with age, followed by standing long jump $(r=-.449)$, modified sit-ups ( $r=-.381)$, and 20-m PACER $(r=-.373$; Table 3). In the middle-aged female group, all physique and physical fitness variables except weight significantly correlated with age, followed by standing long jump ( $r=-.471), 10$-m shuttle run ( $r=-.385)$, and modified situps $(r=-.379$; Table 3$)$

For the old age male group, all physique and physical fitness variables significantly correlated with age, followed by chair standing $(r=-.330)$, figure-of- 8 walk $(r=.322)$, and 3 -m up-and-go $(r=.310$; Table 4$)$. Lastly, for the old age female group, all physique and physical fitness variables except the 6-minute walk test significantly correlated with age, followed

Table 1. Physique and physical fitness characteristics of participants

\begin{tabular}{|c|c|c|c|c|c|c|}
\hline & \multicolumn{2}{|c|}{ Young } & \multicolumn{2}{|c|}{ Middle-aged } & \multicolumn{2}{|c|}{ Older } \\
\hline & $\begin{array}{c}\text { Male } \\
(n=18,772)\end{array}$ & $\begin{array}{c}\text { Female } \\
(n=19,379)\end{array}$ & $\begin{array}{c}\text { Male } \\
(n=13,889)\end{array}$ & $\begin{array}{c}\text { Female } \\
(n=34,260)\end{array}$ & $\begin{array}{c}\text { Male } \\
(n=12,155)\end{array}$ & $\begin{array}{c}\text { Female } \\
(n=24,387)\end{array}$ \\
\hline & $M \pm S D$ & $M \pm S D$ & $M \pm S D$ & $M \pm S D$ & $M \pm S D$ & $\mathrm{M} \pm \mathrm{SD}$ \\
\hline Height (cm) & $174.2 \pm 6.02$ & $161.3 \pm 5.35$ & $169.4 \pm 6.15$ & $157.2 \pm 5.19$ & $165.0 \pm 6.16$ & $152.3 \pm 5.55$ \\
\hline Weight (kg) & $72.4 \pm 11.03$ & $57.0 \pm 8.98$ & $70.9 \pm 9.23$ & $58.7 \pm 7.90$ & $65.5 \pm 8.84$ & $57.4 \pm 7.91$ \\
\hline BMI & $23.8 \pm 3.23$ & $21.9 \pm 3.23$ & $24.6 \pm 2.72$ & $23.7 \pm 2.99$ & $24.0 \pm 2.75$ & $24.6 \pm 3.02$ \\
\hline 10-m shuttle run (s) & $11.1 \pm 1.82$ & $13.5 \pm 1.84$ & $13.1 \pm 2.63$ & $15.2 \pm 2.66$ & - & - \\
\hline 20-m PACER (rep) & $45.5 \pm 19.71$ & $22.4 \pm 11.14$ & $27.9 \pm 14.91$ & $15.3 \pm 8.65$ & - & - \\
\hline Modified sit-ups (rep) & $45.2 \pm 12.22$ & $27.3 \pm 11.65$ & $32.4 \pm 11.96$ & $18.7 \pm 11.27$ & - & - \\
\hline Standing long jump (cm) & $209.3 \pm 30.02$ & $145.9 \pm 24.59$ & $177.6 \pm 33.31$ & $124.5 \pm 27.58$ & - & - \\
\hline Sit-and-reach $(\mathrm{cm})$ & $11.0 \pm 9.20$ & $14.7 \pm 8.73$ & $9.2 \pm 8.72$ & $15.5 \pm 7.49$ & $4.4 \pm 9.66$ & $13.1 \pm 7.84$ \\
\hline Maximal grip strength (kg) & $43.6 \pm 7.46$ & $25.8 \pm 4.81$ & $41.0 \pm 7.37$ & $25.0 \pm 4.56$ & $32.4 \pm 6.85$ & $20.7 \pm 4.54$ \\
\hline Relative grip strength (kg) & $60.8 \pm 10.43$ & $45.9 \pm 8.61$ & $58.2 \pm 10.29$ & $43.0 \pm 8.24$ & $49.8 \pm 10.28$ & $36.4 \pm 8.12$ \\
\hline Chair standing (rep) & - & - & - & - & $19.3 \pm 6.55$ & $17.1 \pm 5.97$ \\
\hline 3-m up-and-go (s) & - & - & - & - & $6.7 \pm 2.36$ & $7.3 \pm 2.59$ \\
\hline 2-minute step-in-place (rep) & - & - & - & - & $104.0 \pm 28.76$ & $96.8 \pm 32.01$ \\
\hline Figure-of-8 walk (s) & - & - & - & - & $25.0 \pm 9.25$ & $27.7 \pm 11.39$ \\
\hline 6-minute walk test (m) & - & - & - & - & $517.2 \pm 114.37$ & $556.7 \pm 109.37$ \\
\hline
\end{tabular}

M, mean; SD, standard deviation. 
Table 2. Relationship between physique and physical fitness variables of young age groups

\begin{tabular}{|c|c|c|c|c|c|c|c|c|c|c|c|c|}
\hline & & 1 & 2 & 3 & 4 & 5 & 6 & 7 & 8 & 9 & 10 & 11 \\
\hline \multirow[t]{11}{*}{ Young male } & 1 & 1 & & & & & & & & & & \\
\hline & 2 & $-.082^{* *}$ & 1 & & & & & & & & & \\
\hline & 3 & $.169^{* *}$ & $.457^{* *}$ & 1 & & & & & & & & \\
\hline & 4 & $.181^{* *}$ & $-.122^{* *}$ & $.059^{* *}$ & 1 & & & & & & & \\
\hline & 5 & $-.317^{* *}$ & $.107^{* *}$ & $-.159^{* *}$ & $-.454^{* *}$ & 1 & & & & & & \\
\hline & 6 & $-.289 * *$ & $.134^{* *}$ & $-.084^{* *}$ & $-.428^{* *}$ & $.519^{* *}$ & 1 & & & & & \\
\hline & 7 & $-.148^{* *}$ & $.226^{* *}$ & $-.063^{* *}$ & $-.537^{* *}$ & $.418^{* *}$ & $.513^{* *}$ & 1 & & & & \\
\hline & 8 & $-.078^{* *}$ & $-.016^{* *}$ & $-.606^{* *}$ & $-.202^{* *}$ & $.240^{* *}$ & $.299 * *$ & $.354^{* *}$ & 1 & & & \\
\hline & 9 & $.137^{* *}$ & $.342^{* *}$ & $.417^{* *}$ & $-.182^{* *}$ & $.123^{* *}$ & $.233^{* *}$ & $.352^{* *}$ & $.204^{* *}$ & 1 & & \\
\hline & 10 & -.013 & $-.054^{* *}$ & $-.419^{* *}$ & $-.224^{* *}$ & $.245^{* *}$ & $.289^{* *}$ & $.391^{* *}$ & $.242^{* *}$ & $.633^{* *}$ & 1 & \\
\hline & 11 & $.210^{* *}$ & -.004 & $.805^{* *}$ & $.113^{* *}$ & $-.210^{* *}$ & $-.140^{* *}$ & $-.170^{* *}$ & $-.052^{* *}$ & $.268^{* *}$ & $-.418^{* *}$ & 1 \\
\hline \multirow[t]{11}{*}{ Young female } & 1 & 1 & & & & & & & & & & \\
\hline & 2 & $-.106^{* *}$ & 1 & & & & & & & & & \\
\hline & 3 & $.123^{* *}$ & $.347^{* *}$ & 1 & & & & & & & & \\
\hline & 4 & $.159^{* *}$ & $-.111^{* *}$ & $.082^{* *}$ & 1 & & & & & & & \\
\hline & 5 & $-.235^{* *}$ & $.107^{* *}$ & $-.135^{* *}$ & $-.447^{* *}$ & 1 & & & & & & \\
\hline & 6 & $-.204^{* *}$ & $.103^{* *}$ & $-.092^{* *}$ & $-.416^{* *}$ & $.509^{* *}$ & 1 & & & & & \\
\hline & 7 & $-.099 * *$ & $.164^{* *}$ & $-.097^{* *}$ & $-.525^{* *}$ & $.455^{* *}$ & $.500^{* *}$ & 1 & & & & \\
\hline & 8 & $-.019^{* *}$ & -.002 & $-.015^{* *}$ & $-.223^{* *}$ & $.254^{* *}$ & $.286^{* *}$ & $.300^{* *}$ & 1 & & & \\
\hline & 9 & $.090^{* *}$ & .229 & $.356^{* *}$ & $-.237^{* *}$ & $.202^{* *}$ & $.288^{* *}$ & $.335^{* *}$ & $.217^{* *}$ & 1 & & \\
\hline & 10 & -.009 & .003 & $-.411^{* *}$ & $-.281^{* *}$ & $.288^{* *}$ & $.339^{* *}$ & $.387^{* *}$ & $.214^{* *}$ & $.687^{* * *}$ & 1 & \\
\hline & 11 & $.182^{* *}$ & $-.084^{* *}$ & $.903^{* *}$ & $.138^{* *}$ & $-.193^{* *}$ & $-.158^{* *}$ & $-.177^{* *}$ & $-.015^{* *}$ & $.240^{* *}$ & $-.441^{* *}$ & 1 \\
\hline
\end{tabular}

1: Age, 2: Height, 3:Weight, 4: 10-m shuttle run, 5: 20-m PACER, 6: Modified sit-ups, 7: Standing long jump, 8: Sit-and-reach,

9: Maximal grip strength, 10: Relative grip strength, 11: BMI

${ }^{* *} p<.01$.

Table 3. Relationship between physique and physical fitness variables of middle age groups

\begin{tabular}{|c|c|c|c|c|c|c|c|c|c|c|c|c|}
\hline & & 1 & 2 & 3 & 4 & 5 & 6 & 7 & 8 & 9 & 10 & 11 \\
\hline \multirow[t]{11}{*}{ Middle male } & 1 & 1 & & & & & & & & & & \\
\hline & 2 & $-.278^{* *}$ & 1 & & & & & & & & & \\
\hline & 3 & $-.183^{* *}$ & $.527^{* *}$ & 1 & & & & & & & & \\
\hline & 4 & $.355^{* *}$ & $-.191^{* *}$ & $-.066^{* *}$ & 1 & & & & & & & \\
\hline & 5 & $-.373^{* *}$ & $.196^{* *}$ & $.033^{* *}$ & $-.496^{* *}$ & 1 & & & & & & \\
\hline & 6 & $-.381^{* *}$ & $.218^{* *}$ & $.049^{* *}$ & $-.466^{* *}$ & $.554^{* *}$ & 1 & & & & & \\
\hline & 7 & $-.449 * *$ & $.323^{* * *}$ & $.103^{* *}$ & $-.628^{* *}$ & $.506^{* *}$ & $.564^{* *}$ & 1 & & & & \\
\hline & 8 & $-.081^{* *}$ & $-.024^{* *}$ & $-.093^{* *}$ & $-.197^{* *}$ & $.224^{* *}$ & $.279^{* *}$ & $.245^{* *}$ & 1 & & & \\
\hline & 9 & $-.330^{* * *}$ & $.450^{* * *}$ & $.397^{* * *}$ & $-.367^{* * *}$ & $.310^{* *}$ & $.423^{* *}$ & $.479^{* *}$ & $.175^{* *}$ & 1 & & \\
\hline & 10 & $-.206^{* *}$ & $.088^{* *}$ & $-.307^{* *}$ & $-.334^{* *}$ & $.342^{* *}$ & $.399^{* *}$ & $.420^{* *}$ & $.239^{* *}$ & $.738^{* *}$ & 1 & \\
\hline & 11 & $-.032^{* *}$ & $-.036^{* *}$ & $.828^{* *}$ & $.043^{* *}$ & $-.163^{* *}$ & $-.081^{* *}$ & $-.087^{* *}$ & $-.096^{* *}$ & $.173^{* *}$ & $-.417^{* *}$ & 1 \\
\hline \multirow[t]{11}{*}{ Middle female } & 1 & 1 & & & & & & & & & & \\
\hline & 2 & $-.271^{* *}$ & 1 & & & & & & & & & \\
\hline & 3 & -.009 & $.364^{* *}$ & 1 & & & & & & & & \\
\hline & 4 & $.385^{* *}$ & $-.166^{* *}$ & $.127^{* *}$ & 1 & & & & & & & \\
\hline & 5 & $-.317^{* *}$ & $.160^{* *}$ & $-.164^{* *}$ & $-.460^{* *}$ & 1 & & & & & & \\
\hline & 6 & $-.379^{* *}$ & $.212^{* *}$ & $-.140^{* *}$ & $-.422^{* *}$ & $.508^{* *}$ & 1 & & & & & \\
\hline & 7 & $-.479 * *$ & $.243^{* *}$ & $-.137^{* *}$ & $-.626^{* *}$ & $.495^{* *}$ & $.524^{* *}$ & 1 & & & & \\
\hline & 8 & $.058^{* *}$ & -.003 & $-.087^{* *}$ & $-.148^{* *}$ & $.200^{* *}$ & $.243^{* *}$ & $.166^{* *}$ & 1 & & & \\
\hline & 9 & $-.238^{* *}$ & $.362^{* *}$ & $.270^{* *}$ & $-.279^{* *}$ & $.263^{* *}$ & $.325^{* *}$ & $.344^{* *}$ & $.180^{* *}$ & 1 & & \\
\hline & 10 & $-.224^{* * *}$ & $.089^{* * *}$ & $-.411^{* * *}$ & $-.342^{* * *}$ & $.360^{* *}$ & $.400^{* *}$ & $.411^{* *}$ & $.219^{* *}$ & $.754^{* *}$ & 1 & \\
\hline & 11 & $.132^{* *}$ & $-.137^{* * *}$ & $.871^{* *}$ & $.223^{* *}$ & $-.258^{* *}$ & $-.261^{* *}$ & $-.274^{* *}$ & $-.080^{* *}$ & $.096^{* *}$ & $-.486^{* *}$ & 1 \\
\hline
\end{tabular}

1: Age, 2: Height, 3:Weight, 4: 10-m shuttle run, 5: 20-m PACER, 6: Modified sit-ups, 7: Standing long jump, 8: Sit-and-reach, 9: Maximal grip strength, 10: Relative grip strength, 11: BMl

${ }^{* *} p<.01$. 
Table 4. Relationship between physique and physical fitness variables of old age groups

\begin{tabular}{|c|c|c|c|c|c|c|c|c|c|c|c|c|c|}
\hline & & 1 & 2 & 3 & 4 & 5 & 6 & 7 & 8 & 9 & 10 & 11 & 12 \\
\hline \multirow[t]{12}{*}{ Old male } & 1 & 1 & & & & & & & & & & & \\
\hline & 2 & $-.171^{* *}$ & 1 & & & & & & & & & & \\
\hline & 3 & $-.167^{* *}$ & $.530^{* *}$ & 1 & & & & & & & & & \\
\hline & 4 & $-.330^{* *}$ & $.086^{* *}$ & $.052^{* *}$ & 1 & & & & & & & & \\
\hline & 5 & $-.224^{* *}$ & $-.046^{* *}$ & $-.039 * *$ & $.300^{* *}$ & 1 & & & & & & & \\
\hline & 6 & $.310^{* *}$ & $.134^{* *}$ & $-.073^{* *}$ & $-.502^{* *}$ & $-.209^{* *}$ & 1 & & & & & & \\
\hline & 7 & $-.268^{* *}$ & $.150^{* *}$ & $.101^{* *}$ & $.518^{* *}$ & $.223^{* *}$ & $-.477^{* *}$ & 1 & & & & & \\
\hline & 8 & $.322^{* *}$ & $-.128^{* *}$ & $-.066^{* *}$ & $-.482^{* * *}$ & $-.227^{* *}$ & $.565^{* *}$ & $-.453^{* *}$ & 1 & & & & \\
\hline & 9 & $.083^{* *}$ & -.070 & -.038 & -.015 & -.041 & .023 & $-.095^{* *}$ & $.306^{* *}$ & 1 & & & \\
\hline & 10 & $-.371^{* *}$ & $.429^{* *}$ & $.377^{* *}$ & $.386^{* *}$ & $.207^{* *}$ & $-.345^{* *}$ & $.326^{* *}$ & $-.304^{* *}$ & .011 & 1 & & \\
\hline & 11 & $-.275^{* *}$ & $.112^{* *}$ & $-.249 * *$ & $.364^{* *}$ & $.231^{* *}$ & $-.313^{* *}$ & $.275^{* *}$ & $-.275^{* *}$ & .039 & .790 & 1 & \\
\hline & 12 & $-.087^{* *}$ & $-.027^{* *}$ & $.830^{* *}$ & .006 & -.015 & .000 & $.021^{* *}$ & .005 & .006 & .164 & -.366 & 1 \\
\hline \multirow[t]{12}{*}{ Old female } & 1 & 1 & & & & & & & & & & & \\
\hline & 2 & $-.333^{* *}$ & 1 & & & & & & & & & & \\
\hline & 3 & $-.204^{* *}$ & $.466^{* *}$ & 1 & & & & & & & & & \\
\hline & 4 & $-.376^{* *}$ & $.135^{* *}$ & $-.016^{* *}$ & 1 & & & & & & & & \\
\hline & 5 & $-.278^{* *}$ & $.120^{* *}$ & $.021^{* *}$ & $.329^{* *}$ & 1 & & & & & & & \\
\hline & 6 & $.435^{* *}$ & $-.247^{* * *}$ & $-.052^{* *}$ & $-.547^{* *}$ & $-.309^{* *}$ & 1 & & & & & & \\
\hline & 7 & $-.373^{* *}$ & $.241^{* *}$ & $.072^{* *}$ & $.526^{* *}$ & $.285^{* *}$ & $-.522^{* *}$ & 1 & & & & & \\
\hline & 8 & $.447^{* *}$ & $-.243^{* *}$ & $-.062^{* *}$ & $-.508^{* *}$ & $-.280^{* *}$ & $.641^{* *}$ & $-.508^{* *}$ & 1 & & & & \\
\hline & 9 & -.003 & -.011 & .019 & -.016 & -.003 & .047 & -.011 & $.070^{* *}$ & 1 & & & \\
\hline & 10 & $-.371^{* *}$ & $.406^{* *}$ & $.281^{* *}$ & $.340^{* * *}$ & $.278^{* * *}$ & $-.350^{* *}$ & $.335^{* * *}$ & $-.316^{* * *}$ & .010 & 1 & & \\
\hline & 11 & $-.240^{* *}$ & $.109^{* *}$ & $-.328^{* * *}$ & $.339^{* *}$ & $.258^{* *}$ & $-.307^{* *}$ & $.280^{* *}$ & $-.270^{* *}$ & .004 & $.799 * *$ & 1 & \\
\hline & 12 & $-.039 * *$ & $-.065^{* *}$ & $.850^{* *}$ & $-.093^{* *}$ & $-.042^{* *}$ & $.083^{* *}$ & $-.059 * *$ & $.069^{* *}$ & .026 & .077 & -.433 & 1 \\
\hline
\end{tabular}

${ }^{* *} p<.01$.

by the figure-of- 8 walk ( $r=.447), 3$-m up-and-go $(r=.435)$, and maximal grip strength $(r=.371$; Table 4$)$.

\section{Prediction equations of physical fitness age}

To develop the PFA estimation formulas for Korean adults, this study conducted stepwise regression analysis with physique and physical fitness variables as independent variables and age as the dependent variable (Table 5)

The prediction equations for PFA by sex and age group were selected by considering practicality and predictive accuracy through expert meetings, resulting in the following equations:

(1) PFA for young males $=22.321-.088(20-\mathrm{m}$ PACER $)+.317$ (BMI)

(2) PFA for young females $=24.486-.143(20-\mathrm{m}$ PACER $)+.304$ (BMI)

(3) PFA for middle-aged males $=66.644-.044$ (standing long jump).069 (20-m PACER)-.201 (weight)-.075 (modified sit-ups)+.269 (10$\mathrm{m}$ shuttle run)+.320 (BMI)

(4) PFA for middle-aged females $=66.814-.098$ (standing long jump).113 (modified sit-ups)
(5) PFA for older males $=84.795+.093$ (figure-of- 8 walk)-.100 (chair standing)-.122 (weight)-.102 (relative grip strength)-.060 (sit-andreach)+.147 (3-m up-and-go)

(6) PFA for older females $=80.577+.097$ (figure-of -8 walk) +.306 (3-m upand-go)-.280 (weight)-.088 (relative grip strength)-.069 (sit-andreach)+.393 (BMI)-.088 (chair standing)-.011 (2-minute step-in-place)

\section{Validity analysis of the PFA equations}

To validate the results, $25 \%$ of the subjects were randomly sampled to estimate PFA, and a correlation coefficient with actual age was obtained. The correlation between PFA and actual age was significant in all the groups: young male $(r=.366, p<.001)$, young female $(r=.240, p<.001)$, middle-aged male $(r=.509, p<.001)$, middle-aged female $(r=.493, p<$ $.001)$, older male $(r=.500, p<.001)$, and older female $(r=.570, p<.001)$. In addition, the observed and expected accumulative probability $(\mathrm{P})$ distributions shown in the P-P plot of the standardized residuals of the regression were almost diagonal (Fig. 2), indicating that the prediction equations were valid. 
Table 5. Physical fitness age regression equation for Korean adults according to sex and age group

\begin{tabular}{|c|c|c|c|c|c|c|c|c|}
\hline & & B & SE & $\beta$ & $t$ & $p$ & $R^{2}$ & SEE \\
\hline \multirow[t]{3}{*}{ Young male } & (Constant) & 22.321 & .388 & & 57.600 & $<.001$ & .132 & 5.63 \\
\hline & 20-m PACER & -.088 & .002 & -.283 & -36.702 & $<.001$ & & \\
\hline & $\mathrm{BMI}$ & .317 & .014 & .169 & 21.938 & $<.001$ & & \\
\hline \multirow[t]{3}{*}{ Young female } & (Constant) & 24.486 & .399 & & 61.419 & $<.001$ & .083 & 6.71 \\
\hline & 20-m PACER & -.143 & .005 & -.222 & -29.037 & $<.001$ & & \\
\hline & $\mathrm{BMI}$ & .304 & .016 & .143 & 18.663 & $<.001$ & & \\
\hline \multirow[t]{7}{*}{ Middle male } & (Constant) & 66.644 & 1.017 & & 65.506 & $<.001$ & .260 & 6.24 \\
\hline & Standing long jump & -.044 & .003 & -.199 & -16.094 & $<.001$ & & \\
\hline & 20-m PACER & -.069 & .005 & -.141 & -12.779 & $<.001$ & & \\
\hline & Weight & -.201 & .013 & -.261 & -15.859 & $<.001$ & & \\
\hline & Modified sit-ups & -.075 & .007 & -.122 & -10.941 & $<.001$ & & \\
\hline & 10-m shuttle run & .269 & .035 & .089 & 7.796 & $<.001$ & & \\
\hline & $\mathrm{BMI}$ & .320 & .044 & .120 & 7.261 & $<.001$ & & \\
\hline \multirow[t]{3}{*}{ Middle female } & (Constant) & 66.814 & .173 & & 386.235 & $<.001$ & .248 & 6.10 \\
\hline & Standing long jump & -.098 & .002 & -.379 & -63.188 & $<.001$ & & \\
\hline & Modified sit-ups & -.133 & .004 & -.181 & -30.17 & $<.001$ & & \\
\hline \multirow[t]{7}{*}{ Old male } & (Constant) & 84.795 & .590 & & 143.809 & $<.001$ & .229 & 4.69 \\
\hline & Figure-of-8 walk & .093 & .007 & .157 & 14.324 & $<.001$ & & \\
\hline & Chair standing & -.100 & .009 & -.121 & -11.097 & $<.001$ & & \\
\hline & Weight & -.122 & .006 & -.201 & -22.020 & $<.001$ & & \\
\hline & Relative grip strength & -.102 & .005 & -.196 & -19.712 & $<.001$ & & \\
\hline & Sit-and-reach & -.060 & .005 & -.110 & -11.892 & $<.001$ & & \\
\hline & 3-m up-and-go & .149 & .025 & .064 & 5.816 & $<.001$ & & \\
\hline \multirow[t]{9}{*}{ Old female } & (Constant) & 8.577 & .451 & & 178.543 & $<.001$ & .328 & 4.60 \\
\hline & Figure-of-8 walk & .097 & .004 & .178 & 23.396 & $<.001$ & & \\
\hline & 3-m up-and-go & .306 & .071 & .139 & 17.637 & $<.001$ & & \\
\hline & Weight & -.280 & .008 & -.394 & -35.904 & $<.001$ & & \\
\hline & Relative grip strength & -.088 & .005 & -.127 & -18.942 & $<.001$ & & \\
\hline & Sit-and-reach & -.069 & .004 & -.096 & -15.835 & $<.001$ & & \\
\hline & $\mathrm{BMI}$ & .393 & .021 & .211 & 18.688 & $<.001$ & & \\
\hline & Chair standing & -.088 & .007 & -.093 & -12.609 & $<.001$ & & \\
\hline & 2-minute step-in-place & -.011 & .001 & -.063 & -8.752 & $<.001$ & & \\
\hline
\end{tabular}

\section{DISCUSSION}

In general, physical fitness tends to decrease with age [32]. However, physical fitness levels and physical age differ according to individual management methods $[33,34]$. Therefore, many people currently pay attention to BA instead of CA, in addition to similar concepts such as cognitive age, PFA, perceived age, work ability index, and the frailty index. Some studies have shown that PFA has a relatively high correlation with BA [6,7], and comparison between CA and PFA is often utilized as a health indicator $[33,34]$.

Several studies have been conducted to estimate PFA. Jeong and Park [17] obtained a physical fitness score formula for 166 elderly males aged 65 to 86 years with the following five measures: walking around two chairs in a figure 8 , grip strength, leg endurance against a wall, one-leg balance with eyes closed, and arm curl. However, the study was limited owing to the small sample size, and the measurement variables they used could not be generalized. Han [18] developed a health-related fitness age equation for 200 adult males using $\mathrm{VO}_{2}$ max, sit and reach, sit-ups, reaction time, vertical jump, and one-leg balance with eyes closed. Nevertheless, the results were limited to general application because of the difficulty in measuring the maximum oxygen intake, which is a laboratory test. Son et al. [19] proposed a physical age estimation algorithm for 3,533 males and 3,467 females aged 11 to 65 years. However, it included teenagers to predict the physical age estimation algorithm for adults. LatorreRojas et al. [25] constructed a functional fitness age equation for females aged 50 years and older using a 30-seconds chair stand test, arm curl test, 2-minutes step test, chair sit-and-reach test, and back scratch test. Although general measurement methods were utilized, only females 

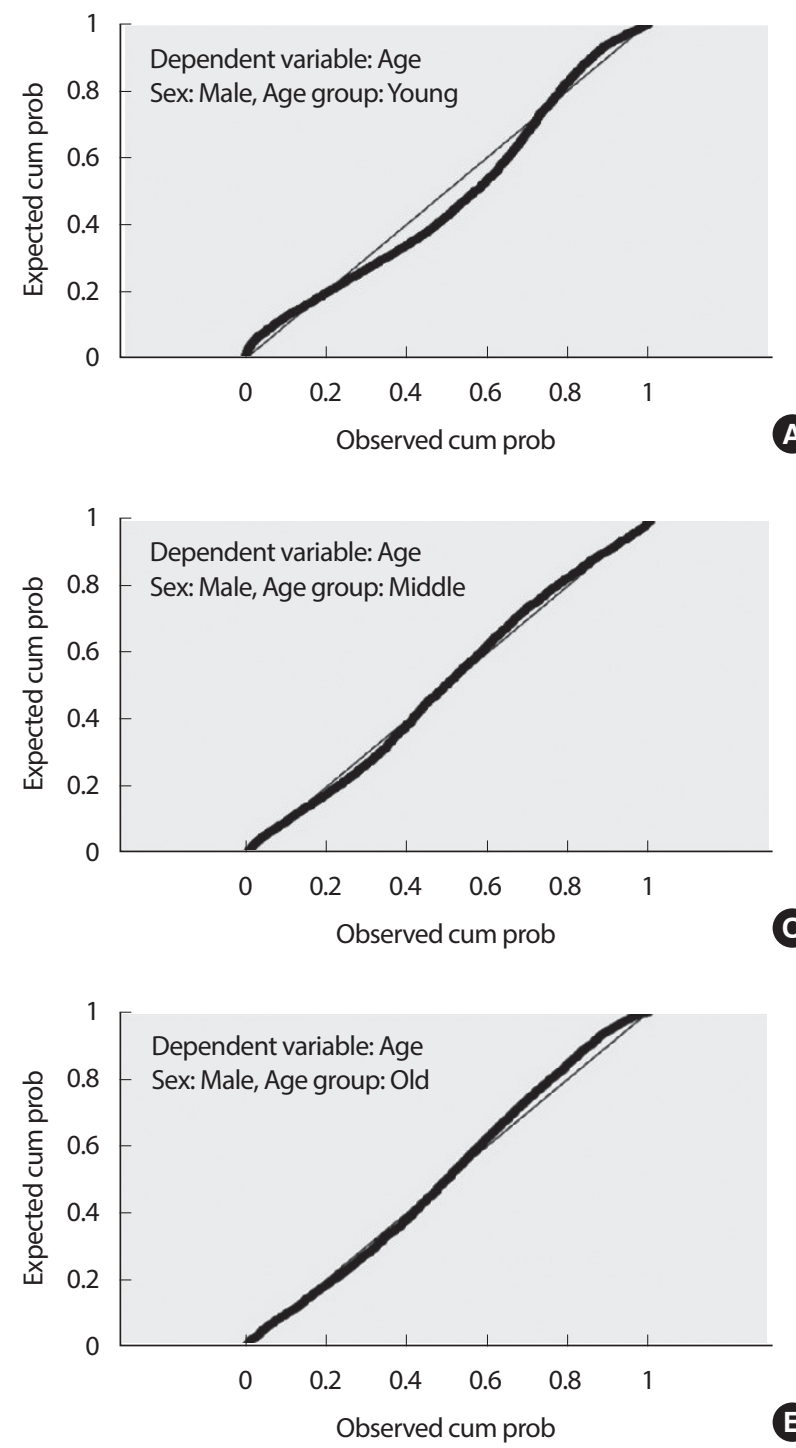
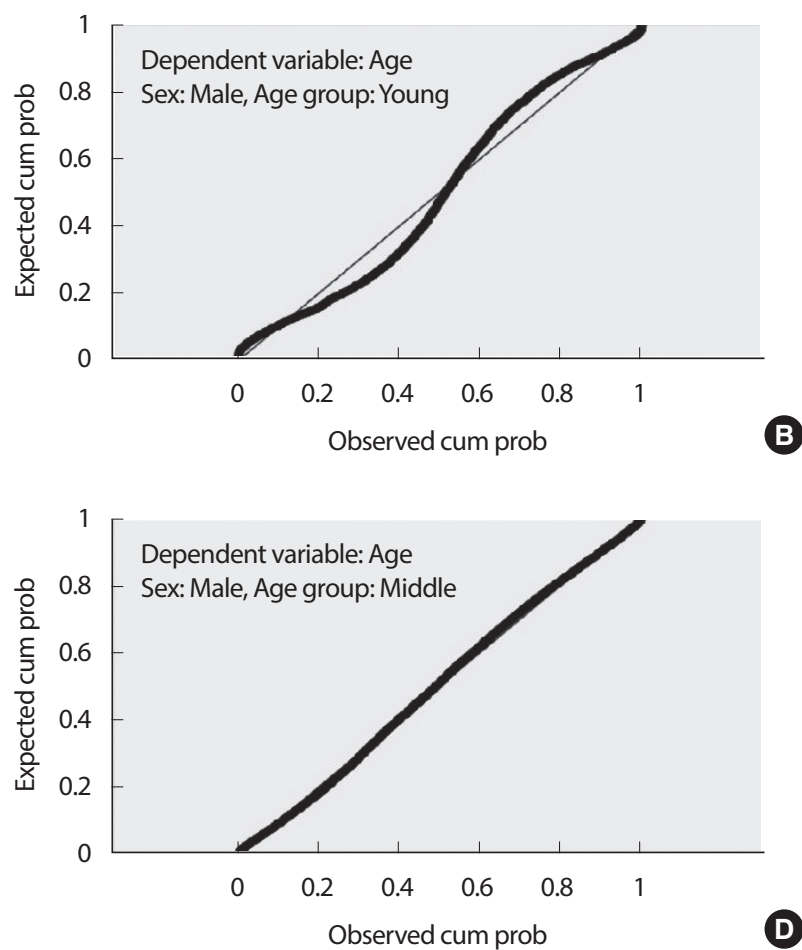

D

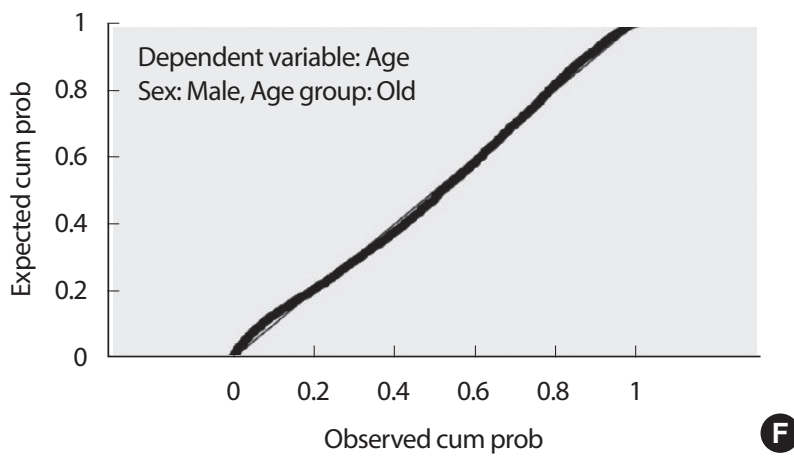

Fig. 2. Normal P-P plot of regression standardized residual of (A) young male, (B) young female, (C) middle-aged male, (D) middle-aged female, (E) older male, and (F) older female.

participated in the study. To compensate for the limitations of previous studies, this study used data from the Survey of National Physical Fitness and National Fitness 100, which included a large number of participants and utilized easily applied tests for data collection. The measurement variables of each group were determined by the studies conducted by the national institution in advance [29-31]. In addition, to reflect the difference by age, we divided the data into 3 age groups; the young (19-40 years), middle (41-64 years), and old (65-80 years) age groups.

To estimate PFA, the strength of the CA correlation to each physical fitness variable should be known [35]. Thus, a correlation analysis was conducted to establish the relationship between age and physical fitness. Afterward, stepwise regression analysis was applied in the development of the prediction equations according to sex and age group, and finally, $25 \%$ of the subjects were randomly sampled to estimate their PFAs for confirming the cross validity of the prediction equations. For the young age groups, 20-m PACER (cardiorespiratory endurance) and BMI were associated with PFA. In the middle age groups, more physical fitness factors affected the PFA in males than in females. In both sexes, standing long jump (power) and modified sit-ups (muscular endurance) influenced PFA, and 20-m PACER (cardiorespiratory endurance), 10-m shuttle run (agility), weight, and BMI were included in the PFA for males. The PFA for older males and females was affected by figure-of- 8 walk (coordination), chair standing (muscular endurance), relative grip strength (muscular strength), sit-and-reach (flexibility), 3-m up-and-go 
(balance), and weight, as well as 2-minute step-in-place (cardiorespiratory endurance) and BMI for females. The older the age group, the more variables affected the estimation of PFA. We consider that this can be interpreted that improving diverse physical fitness factors evenly through various exercises is a way to maintain a younger PFA than CA.

The physiological significance of this study is that the physical fitness level can be evaluated by calculating PFA based on multiple physical fitness measurement results and comparing it with one's CA, rather than by determining high or low through simple scales of single physical fitness variable. However, the study was limited to Korean adults only. Therefore, further studies that develop PFA estimation formulas using data from other countries, especially Western countries, would be meaningful.

\section{CONCLUSION}

In this study, we aimed to develop prediction equations for estimating the PFA of Korean adults in young (19-40 years), middle (41-64 years), and old (65-80 years) age groups. On the basis of the results of this study, we found that different prediction equations should be applied according to age and sex. The evaluation of physical fitness by multiple factors and the physical fitness level by PFA can describe the extent of physical aging and motivate individuals to exercise to improve physical fitness. Therefore, it will be useful to promote physical activity and approach health-related physical fitness maintenance strategy by estimating PFA by each age group and sex. Moreover, our prediction equations for PFA can be used as an excellent tool for prescribing age- and sex-appropriate exercise programs, as well as verifying the effect of the application of the exercise program through the comparison between pre- and post-PFA.

\section{CONFLICT OF INTEREST}

No potential conflict of interest was reported by the authors.

\section{AUTHOR CONTRIBUTIONS}

Conceptualization: B. Sung, W. Song; Data curation: B. Ko, J. Seo; Formal analysis: B. Ko, J. Seo; Funding acquisition: B. Ko; Methodology: B. Ko, J. Seo; Project administration: B. Ko; Visualization: J. Seo; Writing-original draft: B. Ko, J. Seo; Writing-review \& editing: B. Ko, J. Seo, B. Sung, W. Song, J.H. Bae, B. Lim, P. Jamrasi.

\section{ORCID}

$\begin{array}{ll}\text { Byoung-goo Ko } & \text { https://orcid.org/0000-0003-4122-7159 } \\ \text { Ji-won Seo } & \text { https://orcid.org/0000-0003-0279-7562 } \\ \text { Bong-ju Sung } & \text { https://orcid.org/0000-0003-1197-8605 } \\ \text { Wook Song } & \text { https://orcid.org/0000-0002-8825-6259 } \\ \text { Jun Hyun Bae } & \text { https://orcid.org/0000-0003-1918-9213 } \\ \text { Byunggul Lim } & \text { https://orcid.org/0000-0002-4819-7370 } \\ \text { Parivash Jamrasi } & \text { https://orcid.org/0000-0002-5262-8655 }\end{array}$

\section{REFERENCES}

1. Rollandi GA, Chiesa A, Sacchi N, Castagnetta M, Puntoni M, et al. Biological age versus chronological age in the prevention of age associated diseases. OBM Geriat. 2019;3(2):1-14. https://doi.org/10.21926/ obm.geriatr.1902051.

2. Spirduso WW, Francis KL, MacRae PG. Physical dimensions of aging. 2nd ed., Champaign: Human Kinetics 2005.

3. Belsky DW, Caspi A, Houts R, Cohen HJ, Corcoran DL, et al. Quantification of biological aging in young adults. Proc Natl Acad Sci USA. 2015;112(30):E4104-10. https://doi.org/10.1073/pnas.1506264112.

4. Jazwinski SM, Kim S. Examination of the dimensions of biological age. Front Genet. 2019;10:263. https://doi.org/10.3389/fgene.2019. 00263.

5. Harris SE, Deary IJ, MacIntyre A, Lamb KJ, Radhakrishnan K, et al. The association between telomere length, physical health, cognitive ageing, and mortality in non-demented older people. Neurosci Lett. 2006;406(3):260-4. https://doi.org/10.1016/j.neulet.2006.07.055.

6. Nakamura E, Moritani T, Kanetaka A. Biological age versus physical fitness age. Eur J Appl Physiol Occup Physiol. 1989;58(7):778-85. https:// doi.org/10.1007/BF00637391.

7. Nakamura E, Moritani T, Kanetaka A. Biological age versus physical fitness age in women. Eur J Appl Physiol Occup Physiol. 1990;61(3): 202-8. https://doi.org/10.1007/BF00357600.

8. Harnsberger JD, Shrivastav R, Brown WS Jr, Rothman H, Hollien H. Speaking rate and fundamental frequency as speech cues to perceived age. J Voice. 2008;22(1):58-69. https://doi.org/10.1016/j.jvoice.2006. 07.004 .

9. Rippon I, Kneale D, de Oliveira C, Demakakos P, Steptoe A. Perceived age discrimination in older adults. Age Ageing. 2014;43(3):379-86. https://doi.org/10.1093/ageing/aft146. 
10. Cho IH, Park KS, Lim CJ. An empirical comparative study on biological age estimation algorithms with an application of work ability index (WAI). Mech Ageing Dev. 2010;131(2):69-78. https://doi.org/10.1016/ j.mad.2009.12.001.

11. Goggins WB, Woo J, Sham A, Ho SC. Frailty index as a measure of biological age in a Chinese population. J Gerontol A Biol Sci Med Sci. 2005;60(8):1046-51. https://doi.org/10.1093/gerona/60.8.1046.

12. Jones DM, Song X, Rockwood K. Operationalizing a frailty index from a standardized comprehensive geriatric assessment. J Am Geriatr Soc. 2004;52(11):1929-33. https://doi.org/10.1111/j.1532-5415.2004. 52521.x.

13. Searle SD, Mitnitski A, Gahbauer EA, Gill TM, Rockwood K. A standard procedure for creating a frailty index. BMC Geriatr. 2008;8(1):24. https://doi.org/10.1186/1471-2318-8-24.

14. Kim MS. National fitness award 100 in korea. Korean Soc Study of Phys Educ. 2014;19:75-88

15. Esmat T. Fitness for anti-aging. A quarterly publication of the ACSM. 2010:3.

16. Fukunaga TT. Aging effect on life fitness. Japanese J Clin Sports Med. 2004;12(3):377-83.

17. Jeong SB, Park SI. A study on the specification of the estimated formula for physical age of the aged. Korean J Meas Eval Phys Educ Sport Sci. 2000;2:91-106.

18. Han SS. Assessment of health-related fitness for adults. J Korean Phys Educ Assoc Girls Women. 1999;13(2):289-95.

19. Son YS, Bae YJ, Lee DT. Development of physical age estimation algorithm using physical evaluation items. 4th Korean Society of Wellness Autumn Science Congress; Korea. 2011;15-8.

20. Lim BK. A study on regression equation of health-related physical fitness age for male adults. Korea J Growth Dev. 1998;6:1-13.

21. Sung BJ, Ko BG. A study on regression equation of physical fitness age for korean men and women adults. Korean J Meas Eval Phys Educ Sport Sci. 2017;19(2):59-82. https://doi.org/10.21797/ksme.2017. 19.2 .005

22. Lee M, Matsuura Y, Tanaka K. Assessment of physical fitness age in middle-aged and elderly men. Japanese J Phys Fit Sports Med. 1993; 42(1):59-68. https://doi.org/10.7600/jspfsm1949.42.59.

23. Lee MS, Tanaka K, Matsuura Y, Hayakawa Y, Takeda M, et al. Physical fitness age of middle-aged and elderly men with coronary heart disease and its changes following an exercise program. Japanese J Phys Fit Sports Med. 1993;42(4):371-9. https://doi.org/10.7600/jspfsm1949.
42.371

24. Kimura M, Mizuta C, Yamada Y, Okayama Y, Nakamura E. Constructing an index of physical fitness age for Japanese elderly based on 7-year longitudinal data: sex differences in estimated physical fitness age. Age. 2012;34(1):203-14. https://doi.org/10.1007/s11357-011-9225-5.

25. Latorre-Rojas EJ, Prat-Subirana JA, Peirau-Terés X, Mas-Alòs S, Beltrán-Garrido JV, et al. Determination of functional fitness age in women aged 50 and older. J Sport Health Sci. 2019;8(3):267-272. https://doi. org/10.1016/j.jshs.2017.01.010.

26. Kim Y, Austin SB, Subramanian SV, Kawachi I. Body weight perception, disordered weight control behaviors, and depressive symptoms among korean adults: the korea national health and nutrition examination survey 2014. PLoS One. 2018;13(6):e0198841. https://doi.org/ 10.1371/journal.pone.0198841.

27. Pinna F, Murrau R. Age factor and pedestrian speed on sidewalks. Sustainability. 2018;10(11):4084. https://doi.org/10.3390/su10114084.

28. Leleu H, Minvielle E. Relationship between longitudinal continuity of primary care and likelihood of death: analysis of national insurance data. PLoS One. 2013;8(8):e71669. https://doi.org/10.1371/journal. pone.0071669.

29. Korea Institute of Sport Science. The survey of national physical fitness. Seoul, Korea: Ministry of Culture Sports and Tourism; 2009.

30. Korea Institute of Sport Science. Development of National Physical Fitness Certification Standards for Adults. Seoul, Korea: Ministry of Culture, Sports and Tourism; 2010

31. Korea Institute of Sport Science. Development of National Physical Fitness Certification Standards for the Elderly. Seoul, Korea: Ministry of Culture, Sports and Tourism; 2012.

32. Ruiz-Montero PJ, Chiva-Bartoll O, Martín-Moya R. Effects of ageing in physical fitness. Occup Med Health Aff. 2016;4(4):241. https://doi. org/10.4172/2329-6879.1000241

33. Leem KI, Choi DH. A comparative analysis of physical fitness and physique following 7 th physical education curriculum in overweight female high school students. Korean J Health Promot. 2008;8(2):123-31.

34. Williams PT. Physical fitness and activity as separate heart disease risk factors: a meta-analysis. Med Sci Sports Exerc. 2001;33(5):754-61. https://doi.org/10.1097/00005768-200105000-00012.

35. Sato T, Demura S, Murase T, Kobayashi Y. Contribution of physical fitness component to health status in middle-aged and elderly females. J Physiol Anthropol. 2007;26(6):569-77. https://doi.org/10.2114/jpa2. 26.569 . 